\title{
Effects of geometric Berry phase on persistent currents in large- $U$ one-dimensional Hubbard rings
}

\author{
Jian-Xin Zhu and Z. D. Wang \\ Department of Physics, University of Hong Kong, Pokfulam Road, Hong Kong \\ Li Sheng \\ Department of Physics, Nanjing University, Nanjing 210093, People's Republic of China \\ and Department of Physics, University of Hong Kong, Pokfulam Road, Hong Kong
}

(Received 4 May 1995)

\begin{abstract}
By using the Bethe-Yang ansatz within the framework of the tight-binding model, the ground state energy and the persistent current in one-dimensional Hubbard rings are calculated in the presence of an Aharonov-Bohm flux accompanied by a local magnetic field whose direction varies in space. Analytical results are obtained for large- $U$ limit. It is indicated that there usually exists no short periodicity for the ground state energy and persistent current due to the effect of the geometric Berry phase even for the case of infinite $U$. In a special case, the short periodicity retained in the zero-order approximation is broken down by taking into account the first-order energy correction for large but not infinite $U$. Moreover, it is found that in the strong-coupling limit, the electronelectron interaction suppresses the persistent current, which is in agreement with other numerical calculations.
\end{abstract}

Since the discovery of the Berry phase ${ }^{1}$ in 1983 , there has been much interest in the study of topological effects in the fields of quantum mechanics ${ }^{2}$ and mesoscopic physics. ${ }^{3-5}$ The typical example to illustrate the Berry phase is the Aharonov-Bohm (AB) effect ${ }^{6}$ where, even though classical forces are absent, relative phases would accumulate on the wave function of a charged particle passing around opposite sides of a long solenoid due to the presence of a vector potential. Similarly, when a quantum spin follows adiabatically a magnetic field that rotates slowly in time, the spin wave function acquires an additional phase (geometric Berry phase) besides the standard phase (electromagnetic Berry phase) in the static magnetic field. It was shown by Stern ${ }^{7}$ that the geometric Berry phase accumulated on the spin wave function affects the conductance of the ring in a way similar to the $\mathrm{AB}$ effect. Also the Berry phase can be demonstrated in the Aharonov-Casher (AC) effect, ${ }^{8}$ which is an electromagnetic dual of the AB effect-a neutral particle with a magnetic moment may exhibit a topological force-free interference effect, as a result of an interaction with a charged wire. Using a nonperturbative method, Meir, Gefen, and Entin-Wohlman ${ }^{9}$ found that the spin-orbit ( $\mathrm{SO}$ ) interaction induces an additional effective spin-dependent magnetic flux in a mesoscopic ring threaded by a magnetic flux. Aronov and LyandaGeller ${ }^{10}$ demonstrated that the SO interaction results in an SO Berry phase in the adiabatic limit. In the presence of SO interaction, there is a momentum-dependent effective magnetic field coupling to the spin because the Hamiltonian includes a term that is linear in momentum. Therefore, in the adiabatic limit, a geometric Berry phase is acquired by the wave function of the particle under the traversal of the momentum from some initial state through a set of intermediate states in the Hilbert space back to its original configuration. Notice that those studies on effects of geometric Berry phase on persistent currents in mesoscopic rings do not take into account the electron-electron interaction in spite of its importance in some realistic cases. In this paper, by using the Bethe-Yang ansatz within the framework of tight-binding model, which was generalized to the solution of the Hubbard model with twisted boundary conditions by Shastry and Sutherland, ${ }^{11}$ the ground-state energy and the persistent current in one-dimensional (1D) Hubbard rings are calculated in the presence of an $\mathrm{AB}$ flux accompanied by a local magnetic field whose direction varies in space. Analytical results are obtained for large- $U$ limit. It is shown that there usually exists no short periodicity of the ground-state energy and persistent current by introducing the geometric Berry phase even for the case of infinite $U$. As will be seen below, in a special case of $\cos \chi=1$, the short periodicity that is retained in the zero-order approximation is broken down by taking into account the first-order energy correction for large but not infinite $U$. Moreover, it is found that in the strong-coupling limit, the electron-electron interaction suppresses the persistent current, which is in agreement with other numerical calculations.

Let us consider a gas of interacting electrons, confined to a one-dimensional ring of radius $R$. The ring lies in the $x-y$ plane with its center at the origin. A uniform magnetic field $B_{z}$ parallel to the $z$ axis is applied on the arm of the ring while an in-plane magnetic field tangent to the ring $B_{\varphi}$ is induced by a current-carrying wire lying along the $z$ axis. ${ }^{7}$ The tilt angle between two components of the magnetic field is denoted by $\chi=\arctan \left(B_{\varphi} / B_{z}\right)$. The strength of the total magnetic field $B=\sqrt{B_{z}^{2}+B_{\varphi}^{2}}$ is chosen such that the adiabatic approximation is valid but the direct Zeeman contribution to the energy can still be neglected. (This means that the Zeeman energy $\mu_{B} B$ should be much smaller than the Fermi energy $\epsilon_{F}$, but much larger than $\hbar v_{F} / R$ with $v_{F}$ the Fermi velocity, 
which could be simply satisfied. ${ }^{7}$ ) In addition, a solenoid carrying an $\mathrm{AB}$ flux $\Phi_{\mathrm{AB}}$ pierces the hole of the ring. In the context of a tight-binding model, we can treat the system as a closed chain of $N$ sites separated by a distance $a=2 \pi R / N$. The second quantization Hamiltonian of the chain is written as

$$
\begin{gathered}
H=\sum_{l \sigma} \epsilon_{l} c_{l \sigma}^{\dagger} c_{l \sigma} \\
+\sum_{l \sigma} t_{l, l+1}\left(e^{i\left(\theta_{l, l+1}+\lambda_{l, l+1}^{\sigma}\right)} c_{l+1 \sigma}^{\dagger} c_{l \sigma}+\text { H.c. }\right) \\
+U \sum_{l} c_{l \uparrow}^{\dagger} c_{l \uparrow} c_{l \downarrow}^{\dagger} c_{l \downarrow},
\end{gathered}
$$

where $c_{l \sigma}^{\dagger}\left(c_{l \sigma}\right)$ are creation (annihilation) operators for an electron of spin $\sigma$ at the $l$ th site, $\epsilon_{l}$ is the on-site energy, $t_{l, l+1}$ a "bare" nearest-neighbor hopping matrix element, and $U$ the on-site Hubbard repulsion. When electrons hop successively from the $l$ th site to the $(l+1)$ th site, the usual electromagnetic contribution to the phase acquired by electrons is $\theta_{l, l+1}$, which satisfies $\sum_{l} \theta_{l, l+1}=$ $2 \pi \Phi_{\mathrm{AB}} / \Phi_{0}$ with $\Phi_{0}=h c / e$ the flux quantum. In the adiabatic approximation, the spin-dependent geometric contribution due to Zeeman coupling, denoted by $\lambda_{l, l+1}^{\sigma}$, is given by ${ }^{1}$

$$
\lambda_{l, l+1}^{\sigma}=i \int_{l}^{l+1}\left\langle\Pi_{\sigma}(\varphi)|\partial / \partial \varphi| \Pi_{\sigma}(\varphi)\right\rangle d \varphi
$$

where $\left|\Pi_{\sigma}(\varphi)\right\rangle$ is an instantaneous eigenstate of the Hamiltonian $\mathcal{H}(\varphi)=\hat{\boldsymbol{n}}(\varphi) \cdot \boldsymbol{\sigma}$ with $\hat{\boldsymbol{n}}=(-\sin \chi \sin \varphi$, $\sin \chi \cos \varphi, \cos \chi)$ the unit vector along the total magnetic field. We obtain the instantaneous eigenstate

$$
\begin{aligned}
\left|\Pi_{\uparrow}(\varphi)\right\rangle & =\left[\begin{array}{c}
i \cos (\chi / 2) e^{-i \varphi} \\
-\sin (\chi / 2)
\end{array}\right], \\
\left|\Pi_{\downarrow}(\varphi)\right\rangle & =\left[\begin{array}{c}
i \sin (\chi / 2) e^{-i \varphi} \\
\cos (\chi / 2)
\end{array}\right],
\end{aligned}
$$

corresponding to the spin being parallel (antiparallel) to the magnetic field, and find that $\lambda_{l, l+1}^{\sigma}=\left(\varphi_{l+1}-\varphi_{l}\right)(1+$ $\sigma \cos \chi) / 2$ and $\sum_{l} \lambda_{l, l+1}^{\sigma}=\pi(1+\sigma \cos \chi)$.

We now attempt to find the eigenvalues of the Hamiltonian given by Eq. (1). By introducing a unitary transformation

$$
\hat{U}=\prod_{j=1}^{N-1} \exp \left[-i \sum_{\sigma^{\prime}} \sum_{l=j}^{N-1}\left(\theta_{l, l+1}+\lambda_{l, l+1}^{\sigma^{\prime}}\right) c_{j \sigma^{\prime}}^{\dagger} c_{j \sigma^{\prime}}\right]
$$

we can simplify the hopping terms that $t_{l, l+1} \exp \left[ \pm i\left(\theta_{l, l+1}+\lambda_{l, l+1}^{\sigma}\right)\right] \rightarrow t_{l, l+1}(l=$ $1,2, \ldots, N-1) \quad$ while $t_{N 1} \exp \left[ \pm i\left(\theta_{N 1}+\lambda_{N 1}^{\sigma}\right)\right]$ $\rightarrow-t_{N 1} \exp \left[ \pm i\left(2 \pi \Phi_{\mathrm{AB}} / \Phi_{0}+\pi \sigma \cos \chi\right)\right]$. This transformation demonstrates the fact that the flux felt by electrons exhibits a global effect. Because of this, the effect of electromagnetic and geometric phases can be taken into account via the twisted boundary conditions for the wave function

$|\Psi(N+1)\rangle=-\exp [i(2 \pi f+\pi \sigma \cos \chi)]|\Psi(1)\rangle$

where $f=\Phi_{\mathrm{AB}} / \Phi_{0}$. It is quite clear from Eq. (2) that the energy eigenvalue is a periodic function of the magnetic flux with the period $\Phi_{0}$. It is thus sufficient to consider only the range of one $\Phi_{0}$ because the flux can always be reduced by subtracting an integer times $\Phi_{0}$ so that it lies in the specified range. In the following we assume $t_{l, l+1}$ and $\epsilon_{l}$ to be site independent, and set $t_{l, l+1}=-t$ and $\epsilon_{l}=0$. As is well known, if we denote by $N_{e}$ and $M$ the number of electrons and down-spin electrons, the energy eigenfunction is given by the Bethe ansatz in the form ${ }^{11,12}$

$$
|\Psi\rangle=\sum_{1 \leq\left\{x_{i}\right\} \leq N} \sum_{1 \leq\left\{j_{k}\right\} \leq N_{e}} \sum_{\mathcal{P}}\left[\exp \left(i \sum_{n} k_{\mathcal{P} n} x_{n}\right) A\left(\left\{j_{n}\right\} \mid \mathcal{P}\right)\right]\left|\uparrow_{1} \cdots \downarrow_{j_{1}} \cdots \downarrow_{j_{M}} \cdots \uparrow_{N}\right\rangle
$$

imposed by the twisted boundary condition, Eq. (2). Here $\mathcal{P}$ is a permutation of the numbers $\left(1,2, \ldots, N_{e}\right)$ and $A\left(\left\{j_{n}\right\} \mid \mathcal{P}\right)$ the amplitude. The requirement that Eq. (3) should be a solution of the eigenvalue equation $H|\Psi\rangle=E|\Psi\rangle$ leads to

$$
E=-2 t \sum_{n=1}^{N_{e}} \cos \left(k_{n} a\right)
$$

where $k_{n}$ connected with charge waves are determined by the following transcendental equation ${ }^{11}$

$$
\begin{aligned}
k_{n}(N a)= & 2 \pi K_{n}+2 \pi f+\pi \cos \chi \\
& +2 \sum_{j=1}^{M} \arctan \left\{4 t\left[\Gamma_{j}-\sin \left(k_{n} a\right)\right] / U\right\},
\end{aligned}
$$

with the rapidites $\Gamma_{j}$ of spin waves satisfying

$$
\begin{aligned}
& 2 \sum_{n=1}^{N_{e}} \arctan \left\{4 t\left[\Gamma_{j}-\sin \left(k_{n} a\right)\right] / U\right\} \\
& \quad=2 \pi J_{j}-2 \pi \cos \chi+2 \sum_{i=1}^{M} \arctan \left[2 t\left(\Gamma_{j}-\Gamma_{i}\right) / U\right] .
\end{aligned}
$$

Here the constant part of the spin-dependent geometric phase $\pi$ has been absorbed into the first term on the right-hand side of Eq. (5), $K_{n}$ are thus integers (or halfodd integers) for $M$ odd (or even) and $J_{j}$ are integers (or 
half-odd integers) for $N_{e}-M$ odd (or even). In the thermodynamic limit, $k$ and $\Gamma$ are distributed continuously; Eqs. (5) and (6) then lead to the coupled integral equations for distributions, which can be solved more easily. ${ }^{12}$ However, for mesoscopic rings, the finite-size effects must be taken into account carefully, which makes it very difficult to solve the above-mentioned transcendental equations even using numerical methods. Fortunately, when the on-site repulsion $U$ is very strong compared with the hopping amplitude $t$ (i.e., $t / U \ll 1$ ), the ground-state energy and persistent current can be calculated analytically. In the limit of $U / t=\infty$, we are able to arrive at

$$
\begin{array}{r}
k_{n}^{(0)} a=\frac{2 \pi}{N}\left[K_{n}+f+\frac{N_{e}-2 M}{2 N_{e}} \cos \chi+\frac{p}{N_{e}}\right] \\
n=1,2, \ldots, N_{e} .
\end{array}
$$

where $p=\sum_{j=1}^{M} J_{j}$. Obviously, for the case $U=$ $\infty$, the result is quite similar to that for spinless freeelectron mesoscopic rings. The effective flux, which consists of spin-independent $\Phi_{\mathrm{AB}}$, spin-dependent $\left(N_{e}-\right.$ $2 M) \cos \chi / 2 N_{e}$ coming from Zeeman interaction, and $p / N_{e}$ connected with the rapidites $\Gamma_{j}$ of spin waves, determines the eigenenergy by entering the wave vector $k_{n}^{(0)}$, as given by Eq. (7). If $M$ and $p$ are fixed, the system energy can be calculated with consecutive integers (or half-odd integers) of $K_{n}$ that are centered around the origin. The result is given according to the number of electrons $N_{e}$ that are even or odd and itemized below:

(i) $N_{e}=2 m_{1}, M=2 m_{2}$. In this case,

$$
K_{n}=-\left(N_{e}-1\right) / 2, \ldots,\left(N_{e}-1\right) / 2,
$$

and the total energy is found to be

$E^{(0)}=-E_{0} \cos \left[\frac{2 \pi}{N}\left(f+\frac{N_{e}-2 M}{2 N_{e}} \cos \chi+\frac{p}{N_{e}}\right)\right]$,

with $f+\left(N_{e}-2 M\right) \cos \chi / 2 N_{e}+p / N_{e} \in[-1 / 2,1 / 2)$, where $E_{0}=2 t \sin \left(N_{e} \pi / N\right) / \sin (\pi / N)$.

(ii) $N_{e}=2 m_{1}, M=2 m_{2}+1$. We have

$$
K_{n}=-N_{e} / 2, \ldots, N_{e} / 2
$$

and the total energy

$$
E^{(0)}=-E_{0} \cos \left[\frac{2 \pi}{N}\left(f+\frac{N_{e}-2 M}{2 N_{e}} \cos \chi+\frac{p}{N_{e}}-\frac{1}{2}\right)\right],
$$

with $f+\left(N_{e}-2 M\right) \cos \chi / 2 N_{e}+p / N_{e} \in[0,1)$.

(iii) $N_{e}=2 m_{1}+1, M=2 m_{2}$. In this case,

$$
K_{n}=-\left(N_{e}-2\right) / 2, \ldots, N_{e} / 2
$$

and

$E^{(0)}=-E_{0} \cos \left[\frac{2 \pi}{N}\left(f+\frac{N_{e}-2 M}{2 N_{e}} \cos \chi+\frac{p}{N_{e}}+\frac{1}{2}\right)\right]$,

with $f+\left(N_{e}-2 M\right) \cos \chi / 2 N_{e}+p / N_{e} \in[-1,0)$. (iv) $N_{e}=2 m_{1}+1, M=2 m_{2}+1$. In this case,

$$
K_{n}=-\left(N_{e}-1\right) / 2, \ldots,\left(N_{e}-1\right) / 2,
$$

and

$E^{(0)}=-E_{0} \cos \left[\frac{2 \pi}{N}\left(f+\frac{N_{e}-2 M}{2 N_{e}} \cos \chi+\frac{p}{N_{e}}\right)\right]$,

with $f+\left(N_{e}-2 M\right) \cos \chi / 2 N_{e}+p / N_{e} \in[-1 / 2,1 / 2)$.

In the absence of the flux, $J_{j}$ are consecutive integers (or half-odd integers) centered around the origin. When a flux is introduced, the values of $J_{j}$ are shifted to minimize the total energy. In the infinite- $U$ case, the energy spectra versus the flux are identical for different numbers of down-spin electrons, except that there is a relative shift along the flux axis. From the energy favorable point of view, when the magnetic flux varies in the whole range, the number of down-spin electrons is adjusted to arrive at the real ground state with the lowest energy. In the absence of geometric Berry phase, Kusmartsev ${ }^{13}$ found that the ground-state energy of mesoscopic rings with infinite repulsion $U$ varies with the magnetic flux with a surprisingly short period $\Phi_{T}=\Phi_{0} / N_{e}$ by the spin-flip process. In the presence of a spin-dependent geometric phase, there usually exists no short period $\Phi_{T}$. Only in two special cases, $\cos \chi=0,1$, is the short period $\Phi_{T}=\Phi_{0} / N_{e}$ still retained. For the case of $\cos \chi=0$, if we choose $p= \pm M$ for an even number of electrons and $p=0$ for an odd number of electrons, the global energy spectrum can be overlapped completely with that in the absence of the geometric Berry phase by shifting $\Phi_{0} / 2$. For the case of $\cos \chi=1$, we find that the value of $p$ is taken as 0 for an even number of electrons and $M / 2$ for an odd number of electrons so that the energy is periodic in the flux with period $\Phi_{T}=\Phi_{0} / N_{e}$. We plot in Fig. 1(a) the total energy spectrum versus the magnetic flux for a ring of 8 sites and 4 electrons, for various numbers of down-spin electrons and $\cos \chi=1$. The ground-state energy spectrum consists of the lowest segments of all the energy spectra for various $M$. From Fig. 1(a), one can see clearly that the number of down-spin electrons jumps by 1 when the magnetic flux varies over one period $\Phi_{T}$. Thus all possible spin magnetization is experienced by the system when the magnetic flux varies over one flux quantum. Once we have the ground-state energy as a function of the magnetic flux, the persistent current can be obtained straightforwardly by ${ }^{14}$

$$
I=-\frac{e}{2 \pi \hbar} \frac{\partial E}{\partial f}
$$

In Fig. 1(b), we plot the persistent current as a function of $\Phi_{A B}$. It is a piecewise function of the magnetic flux. In each periodic region, it varies linearly with the flux and there are discontinuous jumps when one period is over. For a general value of the tilt angle, the $\Phi_{T}$ periodicity of both the ground-state energy and the corresponding persistent current is absent. As an example, the energy spectrum and the corresponding persistent current with $\cos \chi=0.75$ are plotted in Figs. 2(a) and 2(b), which 
shows the absence of $\Phi_{T}$ periodicity. Comparing Fig. 2(a) with Fig. 1(a), one can also see clearly that as $\cos \chi$ increases, the energy spectra with $M=0,4$ become more close, while the pair with $M=1,3$ become more remote. When $\cos \chi=1$, the two energy spectra for $M=0,4$ overlap completely and the $\Phi_{T}$ periodicity is recovered.

In physically interesting situations, the on-site repulsion $U$ is large but not infinite. Performing the $\sin \left(k_{n} a\right) t / U$ expansion in the Bethe ansatz equations to the first order of $t / U$, we find the correction to the charge momentum as
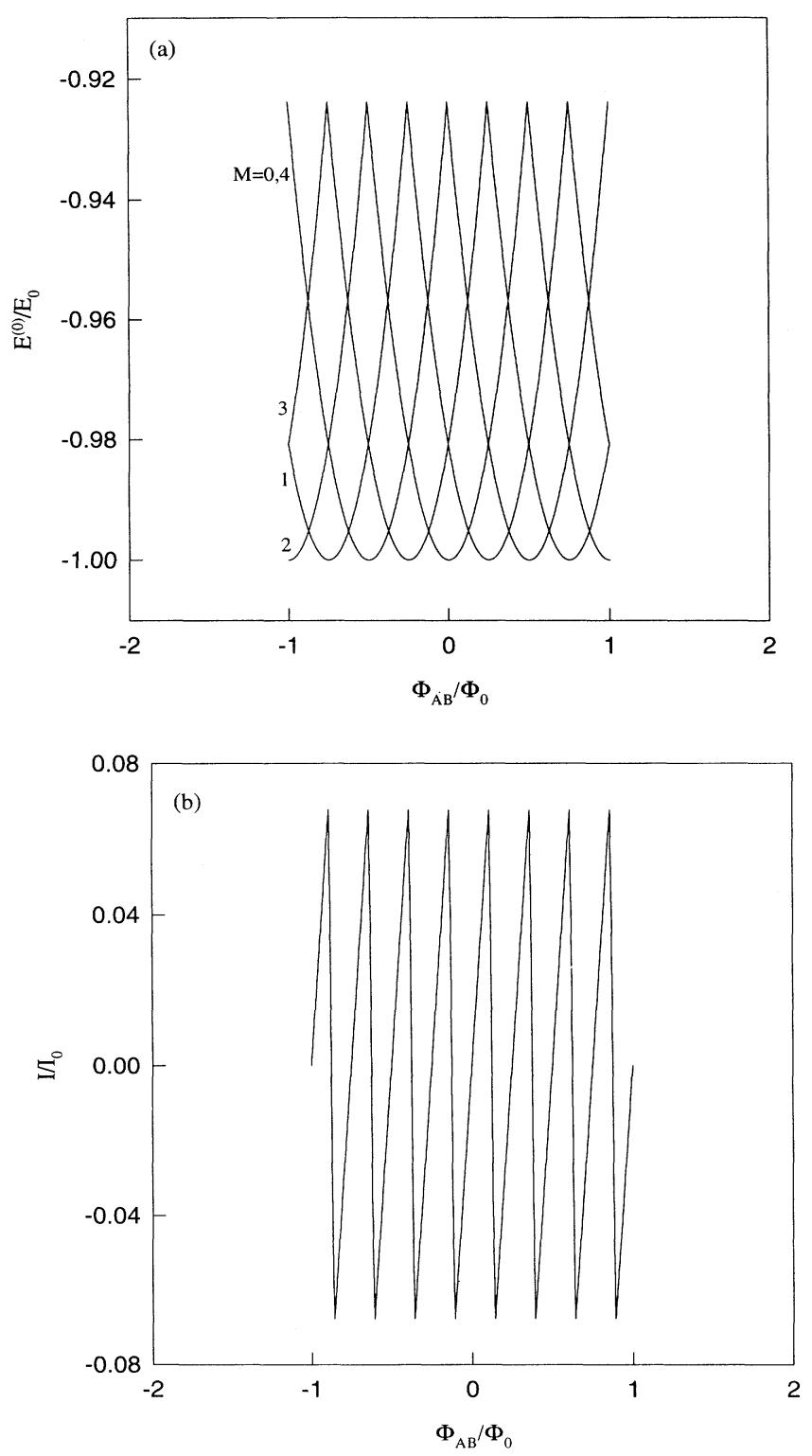

FIG. 1. (a) The total energy and (b) the persistent current as a function of the magnetic flux for a ring of 8 sites and 4 electrons for different number of down-spin electrons, for the case $\cos \chi=1$. $E_{0}=2 t \sin \left(N_{e} \pi / N\right) / \sin (\pi / N)$ and $I_{0}=E_{0} e / 2 \pi \hbar$.

$$
\begin{aligned}
\delta k_{n} a= & -\frac{4 t^{2}}{N U}\left(\sin \left(k_{n}^{(0)} a\right)-\frac{1}{N_{e}} \sum_{n^{\prime}=1}^{N_{e}} \sin \left(k_{n^{\prime}}^{(0)} a\right)\right) \\
& \times \sum_{j=1}^{M} \frac{1}{1 / 4+x_{j}^{2}},
\end{aligned}
$$

where $x_{j}=2 t \Gamma_{j} / U$ are determined by

$$
\begin{aligned}
2 N_{e} \arctan \left(2 x_{j}\right)= & 2 \pi J_{j}-2 \pi \cos \chi \\
& +2 \sum_{i=1}^{M} \arctan \left(x_{j}-x_{i}\right) .
\end{aligned}
$$

By substituting Eq. (13) with the solution of Eq. (14)
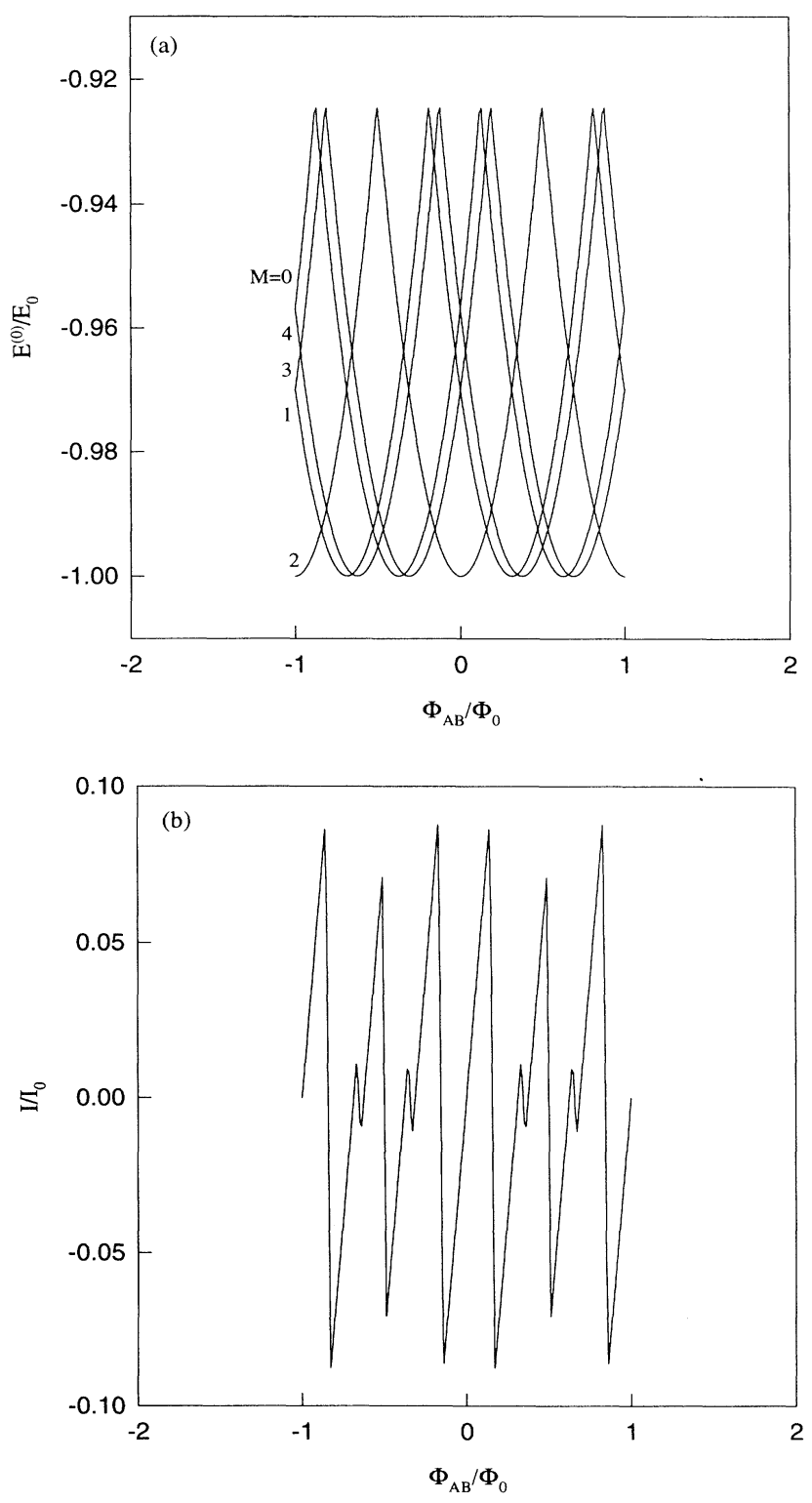

FIG. 2. (a) The total energy and (b) the persistent current as a function of the magnetic flux for the case $\cos \chi=0.75$. The values of other parameters are the same as those in Fig. 1. 
into Eq. (4), the energy correction is obtained

$$
\begin{aligned}
\delta E= & -\frac{4 t^{2} N_{e}}{N U}\left[\sum_{n=1}^{N_{e}} \sin ^{2}\left(k_{n}^{(0)} a\right)-\frac{1}{N_{e}}\left(\sum_{n=1}^{N_{e}} \sin \left(k_{n}^{(0)} a\right)\right)^{2}\right] \\
& \times \sum_{i=1}^{M} \frac{1}{1 / 4+x_{i}^{2}} \\
= & -J \sum_{i=1}^{M} \frac{1}{1 / 4+x_{i}^{2}} .
\end{aligned}
$$

Equations (14) and (15) are quite similar to those for 1D antiferromagnetic Heisenberg problems. It is worth pointing out that the geometric Berry phase now affects the energy corrections by deforming the solutions of Eq. (14). Consider a state for $N_{e}=4$ with $M=$
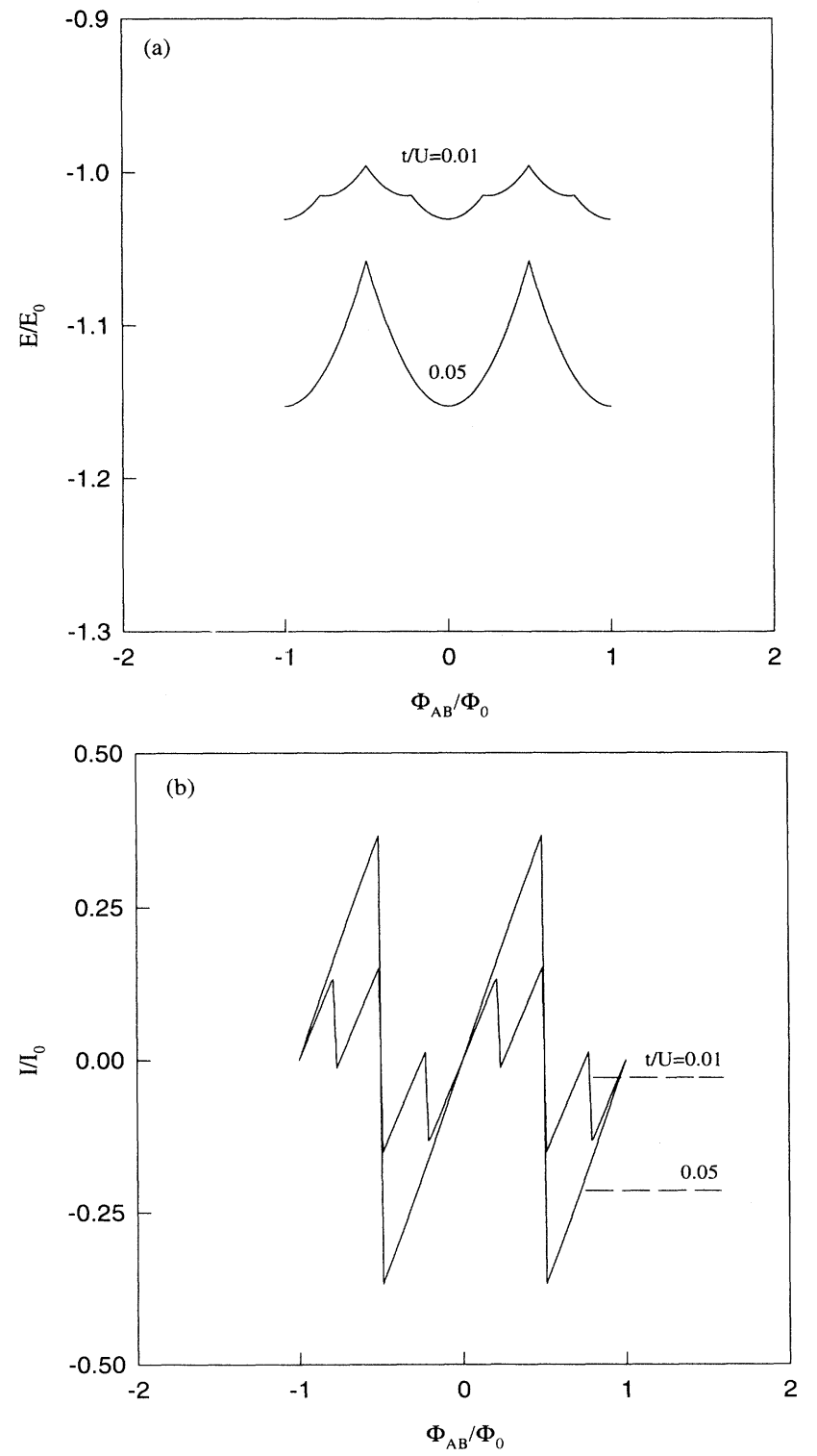

FIG. 3. (a) The ground-state energy and (b) the persistent current as a function of the magnetic flux for $\cos \chi=1$ and $t / U=0.05,0.01$. The values of other parameters are the same as those in Fig. 1.
2, which has the quantum numbers taking on value $J_{1,2}=-1 / 2,1 / 2$ in the absence of Berry phase. We find $\left.\delta E\right|_{M=2}=-6 J$. We now turn on the Berry phase and, as a limiting case, take $\cos \chi=1$. As a result, the previous solution $\left.\delta E\right|_{M=2}=-6 J$ is now deformed to $\left.\delta E\right|_{M=2}=-4 J$. If we concentrated only on the solutions of the Heisenberg model, the ground-state energy would still take the value of $-6 J$ because one can set $J_{1,2}=1 / 2,3 / 2$ to minimize the energy. However, $\delta E$ is now merely the first-order correction to the energy for the $U=\infty$ case. It is thus reasonable, especially for sufficiently large $U$, to consider that the values of $J_{j}$ are determined in the zero-order calculation. In the case of $\cos \chi=1$, the energy corrections for $M=1,3$ are also found, $\left.\delta E\right|_{M=1}=\left.\delta E\right|_{M=3}=-2 J$. (For $\cos \chi=0$, $\left.\delta E\right|_{M=1}=\left.\delta E\right|_{M=3}=-4 J$.) It is evident that the amplitude of the first-order energy correction depends on the number of down-spin electrons. This implies that, in the first-order perturbation, the energy range shared by the energy spectra for different $M$ shrinks and disappears as $U$ decreases. Figure 3 gives the ground-state energy and the corresponding persistent current versus the magnetic flux for a ring of 8 sites and 4 electrons in the case of $\cos \chi=1$. As shown in Fig. 3, as long as the first-order perturbation is employed, the ground-state energy and the persistent current oscillate in the flux with the period of one flux quantum. When $U$ is very large but still not infinite, there are many cusps in the ground-state energy spectrum and correspondingly many jumps in the current over one flux quantum. The number of both cusps and jumps becomes fewer as $U$ becomes smaller. Moreover, this calculation enables us to investigate the influence of the electron-electron interactions on the amplitude of the typical persistent current in the strong-coupling limit. As can be seen in Fig. 3(b), the amplitude becomes smaller as $U$ increases and approaches the limiting value when $U$ is infinite. This observation partly lends support to the numerical calculations by using either the exact diagonalization technique ${ }^{15}$ or the Hartree-Fock approximation, ${ }^{16}$ which show that in the case of a one-dimensional discretelattice ring, the electron-electron interaction suppresses the persistent current.

In summary, we have calculated the ground-state energy and the persistent current in 1D Hubbard rings are calculated in the presence of an $A B$ flux accompanied by a local magnetic field whose direction varies in space. It is indicated that there usually exists no short periodicity for the ground-state energy and persistent current due to the effect of the geometric Berry phase even for the case of infinite $U$. In a special case, the short periodicity retained in the zero-order approximation is broken down by taking into account the first-order energy correction for large but not infinite $U$. Moreover, it is found that in the strong-coupling limit, the electron-electron interaction suppresses the persistent current, which is in agreement with other numerical calculations.

We wish to thank Dr. Qin-Wei Shi for helpful discussions. This work was supported by a RGC research grant of Hong Kong and a CRCG research grant at the University of Hong Kong. 
${ }^{1}$ M. V. Berry, Proc. R. Soc. London Ser. A 392, 45 (1984).

${ }^{2}$ Geometric Phases in Physics, edited by A. Shapere and F. Wilczek (World Scientific, Singapore, 1989).

${ }^{3}$ M. Büttiker, Y. Imry, and R. Landauer, Phys. Lett. 96A, 365 (1983); R. Landauer and M. Büttiker, Phys. Rev. Lett. 54, 2049 (1985); H.-F. Cheung, Y. Gefen, E. K. Riedel, and W.-H. Shih, Phys. Rev. B 37, 6050 (1988).

${ }^{4}$ L. Lévy, G. Dolan, J. Dunsmuir, and H. Bouchiat, Phys. Rev. Lett. 64, 2074 (1990); V. Chandrasekhar, R. A. Webb, M. J. Brady, M. B. Ketchen, W. J. Gallagher, and A. Kleinsasser, ibid. 67, 3578 (1991); D. Mailly, C. Chapelier, and A. Benoit, ibid. 70, 2020 (1993).

${ }^{5}$ Jian-Xin Zhu and Z. D. Wang, J. Phys. Condens. Matter 6, L329 (1994); Phys. Rev. B 50, 7207 (1994); 51, 13813 (1995); Jian-Xin Zhu, Z. D. Wang, and Qin-Wei Shi, J. Phys. A 27, L875 (1994).

${ }^{6}$ Y. Aharonov and D. Bohm, Phys. Rev. 115, 485 (1959).

${ }^{7}$ A. Stern, Phys. Rev. Lett. 68, 1022 (1992).
${ }^{8}$ Y. Aharonov and A. Casher, Phys. Rev. Lett. 53, 319 (1984); C. R. Hagen, ibid. 64, 2347 (1990).

${ }^{9}$ Y. Meir, Y. Gefen, and O. Entin-Wohlman, Phys. Rev. Lett. 63, 798 (1989).

${ }^{10}$ A. G. Aronov and Y. B. Lyanda-Geller, Phys. Rev. Lett. 70, 343 (1993).

${ }^{11}$ B. S. Shastry and B. Sutherland, Phys. Rev. Lett. 65, 243 (1990).

12 E. H. Lieb and F. Y. Wu, Phys. Rev. Lett. 20, 1445 (1968).

${ }^{13}$ F. V. Kusmartsev, J. Phys. Condens. Matter 3, 3199 (1991).

${ }^{14}$ N. Byers and C. N. Yang, Phys. Rev. Lett. 7, 46 (1961); F. Bloch, Phys. Rev. B 2, 109 (1970).

${ }^{15}$ M. Abraham and R. Berkovits, Phys. Rev. Lett. 70, 1509 (1993); G. Bouzerar, D. Poilblanc, and G. Montambaux, Phys. Rev. B 49, 8258 (1994).

${ }^{16}$ H. Kato and D. Yoshioka, Phys. Rev. B 50, 4943 (1994). 